\title{
LEGITIMACIÓN ACTIVA Y REFORMA PROCESAL CIVIL: UNA OPORTUNIDAD
}

\author{
Ius standi and the civil procedure reform: an opportunity
}

Senda Villalobos Indo***

Resumen: El presente artículo aborda la legitimación activa y la importancia de su determinación in limine litis por los tribunales. Para ello, se analizan los distintos conceptos de legitimación activa aceptados doctrinariamente y se estudia la labor atribuida a esta institución dentro del sistema de solución de controversias con relevancia jurídica. Se propone la adopción de un solo concepto de legitimación activa en el futuro Código Procesal Civil, en función de las ventajas que ello presentaría para la administración de justicia en nuestro país.

\begin{abstract}
This article explains the importance of the review in limine litis of the ius standi (legal standing) by courts and analyzes the various concepts of legal standing accepted by the doctrine, as well as the role played by the institution in the controversies resolution system. 'The aim of this work is the proposal of a unique definition of legal standing in the future Civil Procedure Code, based on the advantages that this new concept would provide to the chilean judicial system.
\end{abstract}

Palabras Clave: Reforma procesal civil - Proyecto de reforma procesal civil -Código Procesal Civil - Legitimación activa.

Keywords: Civil procedure reform - Civil procedure reform bill - Civil Procedure Code Legal standing (ius standi).

\section{INTRODUCCIÓN}

\section{a) Antecedentes generales}

La reforma procesal civil, entre otros, se estructura sobre el principio de la descongestión de nuestra judicatura; propósito que ya es posible vislumbrar en el anteproyecto de Código Procesal Civil, elaborado por la Facultad de Derecho de la Universidad de Chile, así como en el Proyecto de Ley que Aprueba el Nuevo Código Procesal Civil. ${ }^{1}$

\footnotetext{
* Abogada, Universidad de Chile. Asociada de Philippi, Yrarrázaval, Pulido \& Brunner. Correo electrónico: svillalobos@philippi.cl.

** El presente artículo se basa en la memoria "La Legitimación procesal Activa y su recepción en el Centro Internacional de Arreglo de Diferencias Relativas a Inversiones, CLADI”, elaborada por Benjamín Mordoj Hutter y Senda Villalobos Indo, para la obtención del grado de Licenciado en Ciencias Jurídicas y Sociales de la Facultad de Derecho de la Universidad de Chile, año 2006.

${ }^{1}$ Boletín 6567-07
} 
Villalobos - Legitimación activa y reforma procesal civil...

Entendiendo lo anterior como un objetivo primordial de los cambios institucionales que se han promovido en el pasado por el legislador (reforma penal, laboral, de familia y tributaria), se hace importante efectuar un análisis de la legitimación activa y su ubicación en el contexto del Derecho Procesal atendiendo a los presupuestos procesales y su correlato con la titularidad del derecho material, al ser éstos los elementos decisivos en la toma de posición para fundamentar la propuesta que se presenta.

El elemento central del asunto está en poder identificar quién tiene la posibilidad de presentar una demanda eficaz, en el sentido de provocar en el órgano jurisdiccional la obligatoriedad de un pronunciamiento sobre el fondo, identificando así al legitimado activo para obrar. Frente a la posibilidad de que se amplíe en demasía o se restrinja arbitrariamente tal legitimación, se hace necesario presentar argumentos tendientes a delimitar a quiénes se otorgará la oportunidad de utilizar los mecanismos de solución de controversias en la nueva judicatura procesal civil.

Luego, la finalidad de proponer un concepto único de Legitimación Activa ${ }^{2}$ (en adelante, indistintamente, "LTA"), en el contexto del Proyecto del futuro Código Procesal Civil, es identificar el vínculo real entre el actor y el titular del derecho invocado, pudiendo así resolverse la cuestión de quién debe hacer valer la pretensión. Logrado lo anterior, se garantizará el efecto de la Cosa Juzgada Sustancial, la cual será estéril en tanto no exista la mencionada correspondencia, toda vez que el verdadero titular del derecho sustancial tendrá la facultad de promover la misma cuestión en un nuevo proceso, lo que generará un gasto de recursos públicos y privados que no es consistente con un sistema eficiente y eficaz de administración de justicia. ${ }^{3}$

\footnotetext{
2 Sólo la Legitimación Activa es pertinente al tema en estudio, por tanto, cada vez que se mencione el concepto de Legitimación sin más calificativo debe entenderse que se usa en este sentido. La Legitimación Pasiva, por su parte es aquella cualidad que debe poder encontrarse en el demandado y que se identifica con el hecho de ser la persona que -conforme a la ley sustancial-está legitimada para discutir u oponerse a la pretensión hecha valer por el demandante en su contra. En razón de lo anterior, es que únicamente a él corresponderá contradecir la pretensión y sólo en su contra se podrá declarar la existencia de la relación sustancial objeto de la demanda. Para un mayor entendimiento consultar: Maturana Miquel, Cristián: Disposiciones Comunes a todo Procedimiento, Universidad de Chile, 2003, pp. 63.

${ }^{3}$ La Cosa Juzgada Sustancial, como posible efecto de ciertas y determinadas resoluciones emanadas de órganos jurisdiccionales, va a surgir una vez alcanzada la inimpugnabilidad de la resolución en el mismo procedimiento (Cosa Juzgada Formal) a lo cual, debe sumarse, su inmutabilidad, esto es, la imposibilidad de reformularlo en cualquier otro juicio en que concurra la triple identidad. Dicha aptitud, va a servir para impedir la eficacia de pretensiones tendientes a cambiar el estado de cosas que - por medio de una sentencia- ha sido reconocido conforme a derecho, como asimismo, permitir se verifique la facultad de poder exigir el cumplimiento en favor del que ha obtenido en el juicio el reconocimiento de su pretensión. No obstante, para lograr el efecto mencionado, es necesario identificar los requisitos exigidos para poder alegar la Cosa Juzgada como excepción, cuando se pretende volver a discutir lo ya resuelto. Así, entre la nueva demanda y la antigua deberá existir exactitud en la identidad legal de personas, en la identidad de la cosa pedida y en la identidad
} 
En suma, la necesidad esencial que cubre la LTA, se despliega ante a la posibilidad de iniciarse un nuevo juicio por quién es, efectivamente, el legitimado activo y, con ello, un nuevo juicio. Por ello, el desafío está en reconocer que si bien, normalmente, la noción de sujeto del proceso, sujeto de la acción y sujeto de la relación sustancial controvertida recaerán en la misma persona, en ciertas ocasiones ello no será así. ${ }^{4}$

\section{b) Importancia de la LTA}

Con el estudio de la LTA se busca establecer si existe un correlato entre el demandante y aquél a quién la ley permite esgrimir la pretensión, debiendo ser el legitimado quien tenga la potestad para afirmar ser titular del derecho material y exigir su satisfacción; es decir, aquella parte capaz de formular la pretensión que se contendrá en la demanda. Así las cosas, se comprende que no se trata de un requisito exigido para obtener una sentencia favorable, sino simplemente para obtener un ejercicio eficaz de la pretensión, en cuanto a obtener un fallo sobre el fondo, que determinará si la pretensión corresponde con la realidad jurídica material.

La LTA es entonces, la posición habilitante para formular la pretensión en condiciones tales que pueda ser examinada por el juez ${ }^{5}$, requiriéndose primero determinar si el actor está o no autorizado por una norma de carácter procesal para pretender. Sólo de ser así, corresponde, en un segundo estadio procesal, establecer si la relación jurídico material existe. Ello supone la posibilidad que la existencia de dicha calidad sea resuelta in limine.

Cuando se exige la legitimación como requisito de un acto -en este caso de la demanda- se parte del supuesto que la concreta relación jurídica no pertenece a cualquiera, sino tan sólo a determinada persona; así, si el poder para obrar es atribuido a alguien en particular, no puede el acto en que tal poder se revela -la demanda- presentar como defecto su ausencia. Sin embargo, no debe pensarse que el derecho a demandar en juicio pertenezca exclusivamente al efectivo titular del derecho material; simplemente bastará el poder tener un derecho, toda vez que el fin de la demanda y el desarrollo del proceso es, precisamente, determinar si a tal posibilidad corresponden los hecho probados en juicio. No obstante, sí es un requisito de legitimación la pertenencia al actor de una determinada situación de hecho, que es la afirmación de la pertenencia del derecho, a la cual, la relación jurídica puede o no corresponder.

de la causa de pedir. El objeto de este examen permitirá determinar si las pretensiones de la demanda son idénticas o no. Para un estudio más acabado ver: Mosquera Ruiz, Mario y Maturana Miquel, Cristian: Breves Nociones acerca de la Cosa Juzgada, 2005.

${ }^{4}$ Ibid, pp. 34-36.

${ }^{5}$ Cfr. Montero Aroca, Juan: La Legitimación en el Proceso Civil, 1994, pp 35. 
De este modo, la reforma al Código Procesal Civil debería propender a que la demanda se presente por quien tiene Legitimatio ad Causam, dado que el demandante tendrá derecho a una sentencia de mérito. Sólo la legitimación de tal naturaleza y con tal efecto pertenecerá a la parte de la litis (parte procesal) la cual sustentará su calidad en presupuestos distintos de quien sostiene ser la parte material, pero a la cual sólo esta calidad (ser parte material) le permitirá de forma eficaz legitimar el ser parte procesal. ${ }^{6}$

Según la doctrina tradicional, si se sujeta la LTA a la determinación de los sujetos facultados para exigir la reparación del perjuicio ocasionado ${ }^{7}$ sólo lo estarán quienes hayan sufrido un menoscabo en su persona o bienes, por lo que la cuestión relevante dirá relación con el objeto tutelado y el titular del derecho. En este escenario, para determinar el sujeto activamente legitimado será obligatorio a identificar el objeto jurídico a tutelar.

El estudio del legitimado -según esta última posición- no se realizará a partir de la identificación de quién es aquel que cuenta con un presupuesto de carácter procesal abstracto -Legitimatio ad Processum - o quién presenta una más particular calidad referente al caso en específico -Legitimatio ad Causam- sino que atenderá a criterios de política legislativa, por fijarse criterios sobre el objeto jurídico a tutelar.

Con todo, frente a la necesidad de encontrar un concepto de LTA que delimite una enorme cantidad de demandas que mermaría el esfuerzo de la reforma procesal civil por entregar una justicia más ágil, no se puede ir en contra de su naturaleza y, menos aún, desconocer arbitrariamente las calidades de que están compuesto, pues se correría el riesgo de excluir a quienes sí favorece. Por el contrario, se debe procurar establecer reglas de LTA que permitan la superación del problema y no la generación de otro más peligroso; cuestión que se generaría si no se fuera consecuente con las directrices de que están compuestos los múltiples aspectos de las instituciones comprometidas.

\section{LOS PRESUPUESTOS PROCESALES Y LA LEGITIMIDAD PARA OBRAR}

Los Presupuestos Procesales (en adelante, "PP") son aquellos antecedentes necesarios que permiten, en primer término, que el juicio tenga existencia jurídica, al ser condiciones mínimas de procesabilidad; y, en segundo lugar, que se alcance su validez formal. ${ }^{8}$

\footnotetext{
${ }^{6}$ Cfr. Carnelutti, Francesco: Instituciones del Proceso Civil, 1956, pp. 467.

7 Cfr. De la Barra Gili, Francisco: Responsabilidad extracontractual por daño ambiental: el problema de la Legitimación Activa, 2002, pp. 267-415.

${ }^{8}$ Cfr. Couture, Eduardo J.: Fundamentos del Derecho Procesal Civil, 1997, pp. 102.
} 
Oskar von Bülow, primer jurista que abordó los PP, sostuvo que son aquellos antecedentes indispensables para lograr tales fines de existencia y validez. Así, es posible distinguir entre éstos a los PP de la acción, de la pretensión, de validez y de una sentencia favorable (aunque esta última categoría ha sido bastante criticada por la doctrina). ${ }^{?}$

Calamandrei, por su parte, señala que los $\mathrm{PP}$ son los requisitos necesarios para que pueda constituirse un procedimiento válido, pues son las condiciones que deben existir a fin de que pueda obtenerse un pronunciamiento cualquiera favorable o no- sobre la demanda, con el objeto de que se concrete el poder/deber del juez de proveer sobre el mérito. ${ }^{10}$

No obstante, la manera en que lo entiende el profesor Cristián Maturana ${ }^{11}$ nos permite determinar un concepto de LTA útil al problema que se plantea en el artículo, siendo necesario esclarecer el escenario del que ésta forma parte, para luego analizar las materias relevantes al efecto. Para ello, resulta de gran utilidad analizar la forma en que agrupa los PP, a saber: (i) PP de Existencia: un juez con jurisdicción, las partes y un conflicto de interés con relevancia jurídica, sin los cuales el proceso nunca logra constituirse; (ii) PP de Validez: normalmente vicios de forma que concurren durante el proceso y que conducen a que éste sea nulo, quedando el juez relevado de dictar sentencia de mérito y que se componen de un Tribunal competente, la capacidad de las partes y el cumplimiento de formalidades legales; (iii) PP para la Eficacia de la Pretensión, Legitimatio ad Causam o Legitimación Procesal: entendiendo que si, por una parte, la Legitimación en la Causa es un presupuesto de eficacia para que en la sentencia exista un pronunciamiento sobre la pretensión hecha valer en la demanda, la correspondencia de la pretensión con la realidad jurídica, por otro lado, es el presupuesto necesario para obtener una sentencia favorable; siendo éste el orden preciso en que deben examinarse los supuestos requeridos. Así, la efectiva concurrencia de los PP de la Pretensión, no dirán relación con la efectividad del derecho como tal, sino con la posibilidad de ejercerlo y de poder afirmar en juicio ser titular del derecho, exigiendo una tutela judicial y permitiendo al juez eximirse de su deber de fallar el fondo; y, (iv) PP de una Sentencia Favorable ${ }^{12-13}$ : equivalentes al hecho de contar con un buen derecho, que facultará al Tribunal a privar a una parte de lo que no es suyo o atribuir a otro lo que le pertenece. Sin embargo, esta categoría no es fiel al correcto entendimiento de la separación entre

\footnotetext{
${ }^{9}$ Cfr. Romero Seguel, Alejandro: El Control de Oficio de los Presupuestos Procesales y la Cosa Juzgada Aparente. La Capacidad Procesal, 2001. pp. 781-789.

${ }^{10}$ Cfr. Calamandrei, Piero: Instituciones de Derecho Procesal Civil, 1962, pp. 350-351.

${ }^{11}$ Cfr. Maturana Miquel, Cristián: Disposiciones (nota 2), pp.117-121.

${ }^{12}$ Cfr. Couture, Eduardo J.: Fundamentos (nota 8), pp. 108.

${ }^{13} \mathrm{Si}$ bien el profesor Cristián Maturana no reconoce en su citado texto esta categoría, por motivos de orden metodológico es pertinente hacernos cargos de ella, en el entendido de que sí es formulada por otros autores como un párrafo adicional.
} 
Villalobos - Legitimación activa y reforma procesal civil...

derecho material -cuya concreción se verifica en la relación jurídica sustancial-y el derecho procesal que se representa en la relación jurídica de las parte en el juicio.

Otra forma de clasificar los PP es distinguir entre los PP de Forma (competencia del juez, capacidad procesal de las partes y demanda en forma) y PP de Fondo (existencia de un derecho tutelado por ley, legitimidad para obrar e interés en obrar) entendiéndose como aquellos requisitos de la sentencia que son necesarios para obtener una resolución que falle sobre la pretensión. Es decir, no se trata de exigencias referentes a la validez del proceso -ya que aún en su ausencia el procedimiento existe y es válido- sino de requisitos para obtener un fallo sobre el fondo, obligando al juez a examinar si el demandante es el verdadero titular de la relación jurídica debatida. ${ }^{14}$

En el siguiente apartado se examinan algunos de los PP mencionados, al resultar pertinentes para demostrar las ventajas de fallar in limine litis la existencia de LTA.

\section{a) La Parte}

El conflicto que se plantea en un juicio enfrenta los intereses ínter subjetivos de las partes, con lo cual, la calidad de parte se determina sobre la base de la posición que se adopta dentro del mismo. Con todo, si bien se es parte por el hecho de participar del conflicto, esta calidad no se alcanza tan sólo por participar en la litis, ya que ser parte abarca aspectos del derecho procesal y del derecho material.

Las partes del proceso son los sujetos de la relación procesal; concepto que se determina en forma diferente al de partes de la relación sustancial, pero que supone, a través de la LTA, el ser (o pretender ser) titular del derecho material invocado. En este sentido, el demandante sustenta su actuación en una pretensión, sin la cual el juez no podrá entrar en el conocimiento del asunto y dicha pretensión sólo podrá ejercerla de manera efectiva el legitimado activo, concluyendo en un fallo que la dirima.

Luego, la importancia de la LTA, en este escenario, radica en su capacidad para vincular la parte material con la parte procesal, contribuyendo a conformar un proceso eficaz si se la entiende y sitúa en una posición que permita delimitar el estudio y fallo de todas las demandas presentadas ante un Tribunal. Tal es la cuestión que se propone recoger en la reforma de nuestra judicatura.

\section{i) La Capacidad de ser Parte}

Carnelutti sostiene que “(...) Es la existencia en el agente de las cualidades necesarias para el ejercicio del poder o para el cumplimiento del deber en que el

\footnotetext{
${ }^{14}$ Cfr. Montero Aroca, Juan: La Legitimación (nota 5), pp. 33.
} 
acto se resuelve". ${ }^{15}$ En conclusión, se es capaz en tanto se pueda ser sujeto de la relación jurídica en que el acto tenga existencia.

Por su parte, la capacidad de obrar-que se exige para incoar un juicio- y la capacidad jurídica-que se identifica con la capacidad de ser parte- son dos aspectos de la misma realidad; es por ello que, cuando la ley procesal exige un cierto modo de ser subjetivo -determinando una capacidad jurídica-, se entiende inmediatamente que la capacidad de obrar es un requisito del acto en que la relación jurídica se desarrolla, pues de nada serviría poder dar nacimiento y participar de ella si posteriormente -al momento de requerir tutela judicial- no se cuenta con los elementos mínimos para obtener el reconocimiento de su existencia y ser su beneficiario.

La doctrina establece que los requisitos necesarios para tener capacidad de ser parte son: ser sujeto de derechos (asimilado a la capacidad de goce) y no estar afectado por alguna incapacidad de ejercicio; de ser así, se es parte a través de un representante legal (equiparado con la capacidad de ejercicio). ${ }^{16}$

Corresponde ahora examinar la capacidad procesal y cómo ésta permite ser parte en la litis, en tanto concepto autónomo que subentiende el tener previamente la capacidad de ser parte.

\section{ii) Capacidad Procesal o Legitimatio ad Processum ${ }^{17}$}

Es la facultad para comparecer en juicio y poder realizar actos procesales con efectos jurídicos. Esto es, poder ser parte en un sentido procesal, exigiéndose su verificación en toda persona que simplemente pretenda poner en marcha el aparato jurisdiccional, sea cual sea su pretensión particular. ${ }^{18} \mathrm{Su}$ concurrencia brinda la posibilidad de actuar en cualquier proceso; a diferencia de lo que ocurre con la LTA, que es una calidad que se requiere para la litis en específico y deben concurrir incluso antes de presentar la demanda -he aquí una de las razones de su homogeneidad-. Lo anterior pues, si bien dicha facultad es de carácter estrictamente procesal, exige encontrarse previamente en posesión de la capacidad en sentido material, facultad ésta que se obtiene fuera del proceso.

En síntesis, atienden a presupuestos de validez, al ser exigencias formales que no miran al proceso individual y subjetivo. Por ello su concurrencia únicamente posibilita la adecuada constitución del juicio y su valida prosecución,

\footnotetext{
${ }^{15}$ Cfr. Carnelutti, Francesco: Instituciones (nota 6), pp. 458.

${ }^{16}$ Cfr. Maturana Miquel, Cristián: Disposiciones (nota 2), pp. 61.

17 La capacidad de obrar se compone de la capacidad para ser parte, capacidad para actuar en el proceso (que de no tenerse se suple actuando a través de un representante) y ius postulandi (capacidad exigida para que la actuación procesal sea correcta y que requiere de constituir patrocinio y poder).

18 Ibid.
} 
Villalobos - Legitimación activa y reforma procesal civil...

sin permitir anticipar el éxito de la pretensión invocada en la demanda, y sin contribuir como elemento restrictivo de demandas que cuenten con un derecho a fallo, como sí lo hace la LTA. Finalmente, la legitimatio ad processum -por su carácter genérico- no bastará para ejercer eficazmente los derechos o deducir determinada pretensión, siendo necesario que la normativa procesal exija reunir una condición más específica referida al juicio determinado, la que provendrá de exhibir legitimación en la causa. ${ }^{19}$

\section{b) La Legitimatio ad Causam, Legitimación Procesal o Legitimidad para Obrar}

"Si la Capacidad es un modo de ser natural del actor, la Legitimatio ad Causam es un modo de ser jurídico". ${ }^{20}$

La doctrina tradicional se encuentra conteste al definir esta cualidad como un requisito necesario para obtener una sentencia de fondo, poniendo el énfasis en el hecho de que sólo aquel que cuente con legitimatio ad causam, tendrá el derecho a exigirle al juez que dirima sobre la pretensión invocada en la demanda; sin embargo, no se aclara quién es el que precisamente cuenta con este derecho o qué elementos o circunstancias permiten adjudicárselo.

Enrique Vescovi, por su parte, define el concepto de forma distinta entendiendo que, si con el nombre de legitimatio ad processum se indica un presupuesto procesal (capacidad de presentarse en juicio por sí o por otros) con la LTA se demuestra la posición de un sujeto respecto al objeto litigioso, lo cual le permite obtener una providencia eficaz. ${ }^{21} \mathrm{El}$ autor recalca la relación material al sostener que la legitimación se obtiene como resultado de participar $-\mathrm{O}$ creer hacerlo, en tanto basta la afirmación de titularidad- de la situación en donde surge o se desarrolla el derecho. De este modo, si el objeto litigioso se identifica con la necesidad de reconocimiento de existencia de un derecho subjetivo o con la comprobación de violación y posterior obtención de tutela del mismo, sólo a quién le es posible exigirla tendrá la calidad de legitimado y, por lo mismo, podrá demandar y pretender tal protección de forma eficaz. ${ }^{22}$

No obstante disentir en algunos aspectos, el criterio reconocido por la mayoría de la doctrina es que la legitimación procesal sería un elemento exigido en relación al juicio en particular, sin que por ello sea necesario ser titular del derecho material para obtenerla, desde que tal exigencia equivaldría en la práctica a obtener siempre un fallo favorable. Con todo, se destaca que sí es indispensable, ser titular

\footnotetext{
${ }^{19}$ Ibid, pp. 63.

${ }^{20}$ Carnelutti, Francesco: Instituciones (nota 6), pp. 465.

21 Cfr. Vescovi, Enrique: “Teoría General del Proceso”, 1984, pp. 197. En Maturana Miquel, Cristián: Disposiciones (nota 2), pp. 64.

${ }^{22}$ En el mismo sentido, Guasp, Jaime: “Derecho Procesal Civil”. 1998. pp. 168. En Maturana Miquel, Cristián: Disposiciones (nota 2), pp. 64.
} 
del interés de que se decida si efectivamente existe o no la relación jurídica sustancial.

En conclusión, por medio del PP de LTA, se exige invocar una pretensión alegando una titularidad, en tanto se puede tener legitimación en la causa sin ser el titular del derecho material pretendido. De acuerdo a lo anterior, la única consecuencia será que la sentencia de fondo desestimará la pretensión absolviendo al demandado. Así las cosas, la legitimatio ad causam puede o no corresponder con una titularidad de derecho subjetivo, siendo perfecta en el caso en que, de existir tal relación, los sujetos del interés en esta declaración sean el demandante y el demandado. Por último, lo expuesto justifica que, en un primer momento y al inicio del proceso, deban examinarse quiénes son los titulares del interés en el litigio.

\section{i) Legitimación Ordinaria}

Es aquella calidad con que se desenvuelve, en el plano procesal, el actor que afirma la existencia de una pretensión material que le corresponde sea satisfecha de acuerdo al derecho sustancial. Para Montero Aroca es "La cualidad de un sujeto jurídico, consistente en hallarse dentro de una situación jurídica determinada, en la posición que fundamenta, según el Derecho, el reconocimiento a su favor de una pretensión que ejercita". ${ }^{23}$

Adentrándonos en la finalidad del artículo es preciso destacar que, cuando se pretende obtener un beneficio jurídico, el proceso tendrá sentido sólo si quien lo inicia es precisamente aquel que ha afirmado ser titular activo de la relación jurídico material. Sin embargo, si la anterior es la situación normal, existen escenarios en que no se puede afirmar tal titularidad, por cuanto ocurren ocasiones que el derecho material simplemente no existe de antemano, siéndose igualmente legitimado activo ordinario. La razón es que, en estos casos, será la ley la que determine qué posición o cualidades debe tener una persona para estar legitimada v.gr. quién es el legitimado para solicitar la interdicción de otra persona. ${ }^{24}$

Siendo de formulación expresa e interpretación restrictiva, lo expuesto reafirma, en primer lugar, la tesis de que la LTA es un PP que opera como límite a la proliferación de procedimientos infructuosos y costosos para el sistema judicial y, en segundo término, la propuesta de este artículo, en lo referido a que nuestros legisladores reconozcan en el futuro Código Procesal Civil esta cualidad, erigiendo a la LTA como una cortapisa que permita al sentenciador, mediante su determinación in limine litis, desestimar una demanda que sólo postula a obtener una sentencia inhibitoria de carácter formal. Por lo demás, precisamente es el legislador procesal el encargado de establecer cuáles serán las posiciones

\footnotetext{
${ }^{23}$ Montero Aroca, Juan: La Legitimación (nota 5), pp. 32.

24 Ibid, pp. 40.
} 
Villalobos - Legitimación activa y reforma procesal civil...

legitimantes, esto es, qué elementos son los que deben concurrir en un sujeto para que pueda de forma eficaz demandar a otro.

$\mathrm{Y}$ es en este punto donde radica el problema, ya que una de estas posiciones legitimantes -que es la de normal ocurrencia- se obtiene simplemente del hecho de asumir ser titular del derecho material, restando protagonismo a la LTA. Sin embargo, puede comprobarse que, en otros contextos, la ley puede llegar a determinar que ni aún en este evento se obtiene legitimación, destacándose su cualidad de límite al desarrollo del procedimiento. Ello ocurre, por ejemplo, en los casos de privación de LTA, como es el caso del fallido en la quiebra, siendo razones de política legislativa las que finalmente conduzcan a establecer a qué sujetos de derecho se les entregará legitimación, en concordancia con aquellas posiciones que desee proteger caso a caso y resguardando los principios a los cuales se les entrega una mayor preponderancia.

\section{ii) Legitimación Extraordinaria}

Es la condición que se confiere -por medio de una norma procesal-a una persona que no afirma como propia la existencia de un derecho material y que - no obstante- se encuentra en una posición legitimante, reconocida jurídicamente. Se asume en el plano normativo, que quien demanda -en estos casos- esgrime una pretensión fundándose en el derecho subjetivo de otro sujeto, que sí es su titular.

En este caso siempre existe una norma expresa que confiere LTA, lo que permite fácilmente al juez comprobar in limine litis su existencia en el demandante, verificando tan sólo si el actor es de aquellos sujetos -distintos de los titulares de la relación jurídica controvertida- que pertenecen a una determinada categoría de interesados en la protección de ciertas situaciones.

De lo señalado hasta ahora es posible colegir que la LTA ordinaria es la que presenta un desafío en el proceso de reforma, por cuanto generalmente se ha sostenido que ésta se obtiene con la sola afirmación de ser titular de un derecho material; lo cual no permite identificar un criterio autosuficiente y capaz de establecer claramente quiénes serán los sujetos que contarán con el derecho a obtener un fallo, es decir, bajo qué criterio se excluye o se permite que sólo ciertas demandas tengan el poder de originar un proceso que culmine con una sentencia con efecto de cosa juzgada sustancial.

En conclusión, la creencia de que aquel que afirme la titularidad de un derecho subjetivo será el legitimado, no permite que la LTA actúe como limitador de demandas, tornándose imprescindible complementarlo con otros elementos que permitan, en su conjunto, obtener la eficiente consecución de un proceso.

\section{iii) Características de la Legitimatio ad Causam}


Como primer punto, reiteramos que ésta no se identifica con el derecho sustancial y sólo requiere para constituirse -en el caso de la LTA ordinaria- de la existencia de una situación de hecho, cual es, la afirmación respecto de la titularidad de una situación jurídica material.

En segundo lugar, no es una condición para el ejercicio de la acción -en tanto el derecho de acción se sustenta en otros presupuestos- sino que es la cualidad necesaria en un sujeto que le permitirá obtener un pronunciamiento sobre la pretensión hecha valer.

Es personal, subjetiva y concreta, pues se debe verificar respecto a un conflicto específico y determinado, en atención a la persona particular del demandante y considerando cuál es el papel que desempeña en el escenario jurídico en que fundamenta su pretensión.

Con todo, lo primordial es que debe existir al tiempo de constituirse la relación procesal, ya que se trata de un PP. En virtud de lo anterior, es pertinente y conveniente que su verificación -de oficio por el juez o a iniciativa del demandado- sea realizada in límine, ya que no tendrá sentido dirimir la pretensión, es decir, la existencia del derecho material, si quien se presenta en la litis como parte, no tiene correlativamente participación en la relación jurídico material, ora como titular, ora ostentando otra posición legitimante.

\section{iv) Naturaleza Procesal de la Legitimatio ad Causam}

Establecer si la LTA es o no una cuestión de fondo o puede ser fallada in limine litis, escapa al plano meramente teórico, siendo su principal consecuencia -en los casos que se alega su inexistencia- su correlato con el establecimiento de la naturaleza del fallo emitido. En esta materia existen dos posturas claramente antagónicas en sus postulados, pero no tan distantes en sus consecuencias prácticas.

En primer lugar, la doctrina más tradicional ${ }^{25}$ sostiene que la LTA se identifica con la titularidad -paradójicamente a determinarse en el juicio- de la situación jurídica controvertida. No se pretende simplemente imponer al actor la necesidad de contar con un PP, sino que se le exige sin más contar con un presupuesto específicamente encaminado a obtener una sentencia favorable, en tanto su comprobación conducirá inevitablemente hacia una protección jurídicamente efectiva. Como consecuencia de lo anterior, se afirma que la resolución que se pronuncia sobre la falta de LTA es una sentencia de mérito, al resolver sobre el fondo sin que pueda fallarse in limine litis.

\footnotetext{
25 De la Oliva sostiene que “(...) Mientras que los temas de fondo, como es la legitimación, condicionan el concreto contenido de tal tipo de sentencias [sentencias sobre el fondo]" En Montero Aroca, Juan: La Legitimación (nota 5), pp. 87.
} 
Villalobos - Legitimación activa y reforma procesal civil...

No obstante, esta tesis contiene contradicciones insalvables, por cuanto, si frente a un fallo que sostuviera la falta de LTA del actor se asumiera que el derecho sustancial no existe y dicha resolución tuviese efecto de cosa juzgada, se estaría resolviendo un asunto de orden material en base a normas de carácter procesal. Sin embargo, ello no ocurre en la práctica, produciéndose, una evidente dicotomía entre el postulado de que la LTA apunta a un tema de fondo y los efectos que se asume tiene la sentencia. Por el contrario, lo que sucede es, simplemente, que el actor o no ha afirmado la titularidad del derecho material o no ha esgrimido la detentación de otra posición legitimante, cuestiones de hecho $-\mathrm{y}$ no de derecho-que finalmente terminan imponiéndose al postulado teórico.

En segundo lugar, si la norma procesal se concibiera en términos tales que facultase a un sujeto a demandar, basándose exclusivamente en el hecho de que éste debe ser el verdadero titular -pues sólo él podría ser la parte procesal- el proceso se construiría sobre la paradoja de que nadie podría demandar en tanto no probara serlo.

Por último, sostener ésta teoría también nos enfrentaría a la imposibilidad de dar cabida a aquellas situaciones en que -por proteger otro tipo de intereses, más allá de los puramente individuales- se le permite también a terceros poder dar inicio a un proceso, es decir, no se permitiría LTA extraordinaria.

La segunda postura surge en el seno de la doctrina moderna. En ella se sostiene que la determinación de la ausencia de LTA - en tanto PP- es materia de una sentencia meramente procesal ${ }^{26}$; en razón de que ésta se regula por normas de Derecho Procesal, por cuanto, es el mismo legislador quien -asumiendo la existencia de diversas posiciones legitimantes- determina las calidades necesarias que debe detentar un sujeto de derecho para poder, eficazmente, esgrimir una pretensión. De esta forma, la sentencia que dirime la alegación de ausencia de legitimación no se funda en normas sustanciales -que sí son pertinentes a la materia a debatirse durante el proceso- sino que lo hace sobre la base de supuestos procesales. Por lo mismo es que, cuando el juez falla sobre la LTA, en ningún caso se está pronunciando sobre la existencia del derecho material, cuestión que debe reservarse para el fondo del litigio.

En síntesis, cuando el juez emite un fallo señalando que el actor no cuenta con LTA, se inhibe de establecer si el actor es o no titular del derecho material, al no abordar el conflicto de intereses surgido entre las partes. Bajo esta postura, las dos únicas situaciones que pueden llevar a un pronunciamiento adverso acerca de la LTA de un litigante se darán en contextos en que, o no se afirme la titularidad del derecho objetivo, o no se asuma ninguna otra posición legitimante, ambas verificables in limine; por lo que no se justifica emprender el desarrollo de un juicio - con todo el desgaste que ello implica- cuando lo que se busca resolver es, simplemente, si el actor es o no la persona que puede eficazmente demandar, y no

${ }^{26}$ Ibid, pp. 87-111. 
determinar si el demandante es o no la persona que cuenta con el específico derecho sustancial que alega.

Finalmente, es importante señalar que la confusión que ha llevado a sostener que la LTA es un tema de fondo ha sido aclarada -gracias a las teorías modernas- al entenderse que, cuando se desarrolla un proceso completo y se desestima la pretensión en la sentencia de mérito -por supuestos motivos de ausencia de LTA- lo que se está indicando es que la LTA con la que contó el actor en un primer momento -pues esgrimió una posición legitimante- no tuvo, finalmente, su correlato en la realidad jurídica que se estableció en la sentencia. Esto es, que si el demandante afirmó tener un derecho material, nunca lo tuvo, siendo posible comprende que un fallo de este tipo, lo que hace es dirimir precisamente sobre la pretensión -desestimándola- por no existir el derecho material.

Al evidenciarse, en razón de lo expuesto, que la LTA es un PP y que, por lo mismo, su ausencia fundamenta fallos meramente inhibitorios, la pregunta de quién es el encargado de controlar su presencia surge naturalmente, como un segundo tema a esclarecer.

Para lograr éste cometido, estamos a lo expuesto por Goldschmidt, al distinguir entre Presupuestos Procesales e Impedimentos Procesales. Así, el citado autor, postula que los primeros deben ser examinados de oficio por el juez, en razón de que su ausencia implica la imposibilidad de dictar un fallo sobre el fondo siendo, precisamente, el juez el encargado de evitar el dispendio procesal que provocaría desarrollar un juicio completo para, finalmente, no dirimir sobre la pretensión alegada. Por su parte, los Impedimentos Procesales -sostiene- deben ser controlados por las mismas partes, ya que su comprobación deriva en su propio beneficio; de esta razón, por lo demás, se hace parte el legislador, cuando les traspasa la carga de alegar su falta. ${ }^{27}$

En suma, el juez no podrá hacer caso omiso del hecho de que el actor no asuma ser titular o no afirme encontrarse dentro de otra posición legitimante únicos dos supuestos de ausencia de LTA- siendo irrelevante, al efecto, que sea el demandado quien alegue tal situación y reforzándose, gracias a ésta teoría, la concepción de que la LTA es un PP.

Otro gran aporte que puede reconocerse, como una secuela de la doctrina impuesta por Goldschmidt, es la conveniencia procesal que se deriva de examinar la concurrencia de la LTA in límine litis. ${ }^{28}$

\footnotetext{
${ }^{27}$ Ibid, pp. 92.

${ }^{28}$ Es pertinente precisar, en este punto, que si bien se entiende que la ausencia de LTA provoca la imposibilidad de la dictación de una sentencia de mérito, la doctrina nacional no se encuentra conteste en señalar cuál es la mejor oportunidad procesal para ser interpuesta una defensa que alegue dicha ausencia y, por lo mismo, cuál es su naturaleza. El reconocer que este vicio del
} 
Villalobos - Legitimación activa y reforma procesal civil...

En este punto, cabe sostener que si finalizado un juicio se dicta un fallo inhibitorio, señalando que el demandante carecía de LTA, el desarrollo del mismo habrá sido en vano y absolutamente evitable; en tanto la comprobación de encontrarse el actor en los supuestos de legitimación podía, y debía realizarse, en los albores del proceso. Por el contrario, si se emite un fallo de mérito y en él se desestima la demanda, no se estará fallando sobre la ausencia de LTA -aunque en la sentencia se recurra a este término- sino que sobre la existencia del derecho material. Tener claro lo anterior es fundamental para esclarecer la confusión que ha conducido a mal entender que la comprobación de la LTA debía, necesariamente, tener un correlato en la titularidad del derecho material, lo cual sería demostrado por un fallo de mérito en el cual se establecería la realidad jurídica sustancial existente entre los litigantes.

\section{c) El Interés para Obrar}

Nace con la finalidad de suplir la necesidad de encontrar un elemento adicional que complemente la afirmación de ser parte de la relación material y que permita identificar quién será legitimado activo; pero sin desconocer que se trata de un concepto que ha sido tradicionalmente tratado como PP de la Pretensión.

Chiovenda señala que este interés “(...) Consiste en una situación de hecho tal, que el actor -sin la declaración judicial- sufriría un daño, de modo que la resolución judicial se presenta como el medio necesario para evitar ese daño". ${ }^{29}$ En este sentido, el análisis apunta a la supuesta necesidad de poseer y reclamar una tutela jurídica -en tanto interés específico e independiente- para conseguir legitimar la actuación del actor, es decir, para tener LTA.

A su turno, Ticona Postigo señala que este tipo de interés envuelve la "Necesidad de tutela jurisdiccional" de una persona en concreto y que lo determina a solicitar, por vía única y sin tener otra alternativa eficaz, la intervención del respectivo órgano jurisdiccional con la finalidad de que resuelva el conflicto de intereses en el cual es parte ${ }^{30}$. Asimismo, Juan Monroy postula que

proceso puede ser resuelto in límine litis y, en consecuencia, darle un tratamiento de excepción dilatoria, permite evitar el desgaste infructuoso que implica el desarrollo completo de un proceso para que, en el momento de dictarse un fallo sobre el fondo, se provoque la situación tal de que, quien interpuso la demanda, no sea aquel que efectivamente poseía interés en tal declaración. Detener el proceso en su umbral permitirá, conjuntamente y sin más demoras, iniciar un nuevo juicio en donde las partes litigantes sean, efectivamente, quienes legítimamente pueden aducir sus pretensiones y contrapretensiones. Para un estudio más acabado sobre la posibilidad de alegar la ausencia de LTA como excepción dilatoria consultar: Figueroa Yávar, Juan Agustín: ¿En qué momento procesal puede alegarse la falta de legitimación activa?, 1997.

${ }^{29}$ Montero Aroca, Juan: La Legitimación (nota 5), pp. 46.

${ }^{30}$ Cfr. Ticona Postigo, Victor: El debido proceso y la demanda civil, 1999, pp. 102. 
existirá interés en obrar cuando se han agotado todos los medios para satisfacer la pretensión material y no se tiene otra alternativa que recurrir al órgano judicial. ${ }^{31}$

En principio, es posible inferir que tendrá interés en obrar exclusivamente el titular del derecho - este último a ser establecido en la sentencia- ya que, existiendo efectivamente éste último, el interés en su tutela lo detentará justamente su titular, el que a su vez contará con LTA, porque habrá invocado la pretensión correspondiente en la demanda. Asimismo, si se comprueba que no existe el derecho sustancial, el legitimado sigue identificándose con quien deseaba la tutela que el ordenamiento finalmente no entregará, puesto que siempre detentó este interés secundario fundándolo -aunque erradamente- en un supuesto interés primario del que se pensaba era titular.

El análisis debe emprenderse, identificando a quién posee un interés primario (que proviene de la relación material) para luego contrastarlo con quién requiere de tutela jurídica en el caso específico, es decir, quién posee un interés secundario. En seguida, sólo aquel que la necesite, buscará obtenerla ejerciendo una acción y para obtenerla fundamentará la pretensión en la afirmación de ser el titular del derecho sustancial, con lo cual, recién el juez podrá adentrarse en su estudio y comprobación a través de los actos en que se desarrolla el proceso.

De esta manera, el juicio concluirá con una sentencia en la cual se precisará si el derecho subjetivo existe o no; permitiendo determinar la parte material, cumpliéndose entonces con el fin de la litis, esto es, componer -al dar prevalencia a uno de ellos- el conflicto de intereses de relevancia jurídica.

Destacamos que el interés en obrar difiere del interés comprometido en el juicio -siendo posterior a éste- pues se trata de un interés en la tutela jurídica del interés primario que surge de la relación jurídica sustancial sobre la cual el juez debe decidir. Es decir, el interés en obrar atiende a la relación jurídica procesal por la cual el juez debe decidir. Sobre el punto Carnelutti señala que “(...) El interés [en obrar] estará constituido por aquello de que sin el proceso la tutela que el ordenamiento jurídico ofrece al interés [primario] del actuante no sería plenamente eficaz".32

Confundir ambos conceptos impide, a su vez, distinguirlos del concepto de LTA, respecto del cual -si bien son independientes- cumplen la tarea de proveerse de recíproco fundamento, ya que cuando el actor no es sujeto de interés en la litis entiéndase interés primario- carece de legitimación; el interés en obrar, por su parte, será un interés adicional que debe presentarse independientemente.

En suma, el interés primario es un presupuesto de fundamentación de la LTA, mientras que la existencia del interés en obrar es un elemento del acto procesal

\footnotetext{
${ }^{31}$ Cfr. Monroy Galvez, Juan: Introducción al Proceso Civil, 1996, pp. 69.

${ }^{32}$ Carnelutti, Francesco: Instituciones (nota 6), pp. 516.
} 
Villalobos - Legitimación activa y reforma procesal civil...

y, por lo tanto, posterior. Es así como se comprende que la legitimación sea preliminar al interés en obrar, interés que sólo puede surgir en quien ya es legitimado. ${ }^{33}$

Finalizando el presente apartado, aclaramos que si bien la necesidad de tutela jurídica se estima por la doctrina como un PP, su efectiva concurrencia no es un presupuesto del proceso, por cuanto ello supondría que únicamente se promoverán litigios fundados, correpondiendo ahora analizar la naturaleza del nexo que une el interés primario con la LTA y qué consecuencia trae consigo la confirmación de su existencia en el proceso.

\section{LA RELACIÓN JURÍDICA MATERIAL Y LA LEGITIMACIÓN ACTIVA}

A diferencia de la capacidad -presupuesto genérico común a cualquier tipo de pretensión de tutela jurídica que se ejercite- la legitimación hace referencia a una determinada relación del sujeto con la situación jurídica sustancial que se deduce en juicio. Lo común será que la relación a que se hace referencia, se concrete en la pretendida detentación de la titularidad de la misma, por parte del actor, pero a veces -sin dejar de presentarse el supuesto de la LTA- no es efectiva tal correlación por estarse ante un caso de legitimación activa extraordinaria.

El análisis de la naturaleza ontológica de la LTA ordinaria dice relación con el examen del derecho sustantivo afectado, en tanto su titularidad abarca la posición legitimante por antonomasia. Sin embargo, existen otras que también sirven de fundamento a la LTA. Así al encontrarse el actor en posesión de una de ellas -ya sea del derecho subjetivo, interés individual, interés supraindividual, interés público u otro- se evidenciaría su legitimación para sostener una determinada pretensión en un proceso.

Por razones de límites en la extensión del presente artículo, a continuación se presentarán brevemente las posiciones legitimantes más corrientes. ${ }^{34}$

\footnotetext{
${ }^{33}$ Ibid.

${ }^{34} \mathrm{El}$ análisis, que sirve de base a este capítulo, es efectuado por Faustino Cordón Moreno en función de proveer una explicación satisfactoria a la existencia de la LTA Extraordinaria. Así, quien la posee, puede derivar su legitimación indirectamente - pues directamente lo hará de la ley-a partir de una de estas posiciones legitimantes.

En el contexto del presente trabajo, en que lo determinante es precisar el fundamento de la LTA Ordinaria, explicaremos dos de estas posiciones, al ser ellas las pertinentes, entendiendo, que cumplen la misma función en la LTA Ordinaria como en la Extraordinaria, puesto que, precisamente, el distanciamiento entre estos conceptos se produce al presentarse como posiciones legitimantes -exclusivamente en la LTA Extraordinaria- el hallarse provisto de un interés supraindividual o de un interés público, por ser aquellos los casos en que la persona que esgrime la pretensión, fundamento de la demanda, no afirma como propia la existencia de relación jurídica material alguna, es decir, no asume ninguna titularidad sustancial.
} 


\section{a) El Derecho Subjetivo}

Habitualmente la iniciación de un proceso quedará supeditada al arbitrio del titular del derecho subjetivo violado o desconocido, siendo éste quien decidirá cuándo es oportuno pretender la defensa de su derecho en juicio; por ello, es que una de las posiciones legitimantes más comunes para formular la pretensión, se basará en afirmar dicha titularidad. ${ }^{35}$

Esta afirmación provee a su turno de fundamento a la legitimación ordinaria, que es aquella que ostenta quien aduce ser el titular de la situación jurídica sustancial deducida en juicio, toda vez que sólo él puede $-\mathrm{y}$ buscaobtener una tutela jurídica.

A su turno, el escenario descrito normalmente ocurre en plano de situaciones jurídicas privadas, donde el derecho subjetivo es de titularidad individual. No obstante, dicho esquema soslaya diferentes realidades consustanciales al ejercicio de ciertas y determinadas acciones- en que no es tanto la relación entre derecho material afectado y la pretensión procesal deducida la que justifica la relación trabada en la litis, sino que es la ley -de forma autónoma- la que, con independencia de los supuestos anteriormente señalados, determina quién tiene legitimación.

\section{b) El Interés Individual o Interés Primario}

Es un concepto distinto del de derecho subjetivo privado, en un sentido estricto. El interés individual surge a propósito de la supuesta existencia de una posición jurídica amenazada, razón por la cual, quien se atribuye dicha posición, exige determinada tutela jurisdiccional. Como no existe una necesidad a ultranza de que la anterior atribución provenga de una relación jurídica sustancial real cuestión que determinará la sentencia- es que se exige, para obtener LTA, que quien ejerza la acción haya a lo menos sufrido un perjuicio o dejado de obtener una ganancia. Todo ello, conforme a un derecho del que se supone titular, configurándose una específica situación jurídica amparada por el ordenamiento.

Sin embargo, cuando la base del sistema se construye sobre la protección de situaciones jurídicas individuales existe la amenaza de que la extensión de la legitimación se vea enfrentada a la amenaza de desvincular el interés individual proveniente de la situación jurídica protegida- del efectivo titular, es decir, aquella persona concreta y determinada que exclusivamente puede de forma eficaz fundamentar la LTA.

En este contexto, la LTA se deriva, ante todo, del hecho de encontrarse en una situación jurídica particular, respecto de la cual el ordenamiento jurídico

\footnotetext{
${ }^{35}$ Cfr. Montero Aroca, Juan: La Legitimación (nota 5), pp. 38.
} 
Villalobos - Legitimación activa y reforma procesal civil...

entrega algún tipo de respaldo normativo; así, el interés legítimo se percibe como "Todo interés individual o socialmente tutelado por el Derecho, indirectamente, con ocasión del interés general, y no configurado como derecho subjetivo (...) y que puede ser impugnado por su titular con independencia de si se le ocasiona de forma efectiva un beneficio o perjuicio [al derecho material]". ${ }^{36}$

Bajo este entendimiento, será legítimo contradictor quien haya sufrido un daño sobre un interés legítimo, es decir, un interés respaldado normativamente, por lo que se deberá determinar quién ha sufrido el daño, ya que éste será el legitimado para ser titular de la acción reparatoria. De esta forma, la existencia del sujeto víctima del daño es un requisito indispensable para que surja la acción de reparación, pues frente a la máxima donde no bay interés no bay acción, la víctima es quien puede perseguir la indemnización, pues ésta es quien se ha visto disminuida -en su persona o bienes- con la actuación de un tercero, siendo por lo mismo acreedora de la obligación de resarcir el daño por el autor de éste.

No obstante, reiteramos que tal concepción no nos provee de una norma de clausura que nos señale qué daño y en qué proporción será suficiente como para poder fundamentar la pretensión. Y el no establecer un límite -y con ello perder una única concepción de LTA, necesaria y suficiente, para ser fallada in limine litis- podría provocar la saturación del aparato jurisdiccional.

\section{LA LEGITIMACIÓN ACTIVA Y SU RECEPCIÓN EN EL PROYECTO DE LEY QUE APRUEBA EL NUEVO CÓDIGO PROCESAL CIVIL}

Adentrarse en la tarea enunciada exige realizar, en forma previa, un breve estudio del anteproyecto de Código Procesal Civil, con el fin de comprobar la evolución que -como señala el Mensaje $\mathrm{N}^{\circ} 398-357^{37}$ - es posible verificar entre la convocatoria efectuada en mayo de 2005, por parte del Ministerio de Justicia a magistrados, académicos, abogados litigantes, personeros del mundo público, representantes de diversas Universidades e intervinientes en el campo del Derecho Procesal, quienes participaron del Foro Procesal Civil, y los resultados finales de dicho proceso con la presentación del Proyecto del Nuevo Código Procesal Civil, en mayo del año 2009, y su actual tramitación en la Cámara de Diputados. ${ }^{38}$

\section{a) El anteproyecto de Código Procesal Civil}

\footnotetext{
${ }^{36}$ Gómez-Ferrer Morant, Rafael: Derecho a la tutela judicial y posición peculiar de los poderes públicos, 1982, pp. 189.

${ }^{37}$ Mensaje de S.E. La Presidenta De La República con el que inicia un proyecto de ley que aprueba el nuevo Código Procesal Civil. Santiago. 18 de mayo de 2009.

38 A la fecha de presentación del artículo, el Proyecto se encuentra en la primera etapa del trámite constitucional, Particularmente, el 29 de septiembre de 2009, se dio Cuenta del Mensaje $\mathrm{N}^{\circ} 1187$ 357 que retira la urgencia simple.
} 
El encargo efectuado por el Ministerio de Justicia, particularmente a la Facultad de Derecho de la Universidad de Chile, finalizó a fines del año 2006 con la entrega de un anteproyecto de Código Procesal Civil. La importancia del documento radica en haber sido la base y guía para la activa y pormenorizada discusión que, en su segunda etapa, el Foro Procesal Civil inició en marzo del año 2007 y que culminó en mayo del año 2009.

En el contexto del análisis abordado en los capítulos anteriores y sobre la base de los mismo, es pertinente destacar que desde un inicio -en los artículos 28 (Partes), 29 (Capacidad para ser partes) y 30 (Capacidad procesal)- es posible colegir que la regulación de esta materia se funda exclusivamente en normas de derecho material, sin atender a las consecuencias que ello trae aparejado si a la vez no se refuerza de manera clara y explícita con un concepto determinado de LTA.

Al respecto, es preciso recalcar que si bien se es parte por el hecho de participar del conflicto, no basta simplemente con intervenir en la litis, ya que ser parte abarca aspectos del derecho material y del derecho procesal.

Por la forma en que han sido concebidos dichos conceptos en el anteproyecto -como un modo de ser subjetivo- debería concederse a la LTA la posibilidad de vincular la parte material con la parte procesal, toda vez que la capacidad para ser parte, o su ausencia (artículo 32), debe necesariamente complementarse con la capacidad procesal (artículo 30) que equivale a la facultad para comparecer en juicio y poder realizar actos procesales con efectos jurídicos.

Lo anterior, es fundamental para alcanzar los fines de la reforma principalmente la descongestión de nuestros Tribunales- por cuanto la capacidad procesal faculta para actuar en cualquier proceso, en cambio la LTA otorga el necesario complemento para obtener la calidad particular que se requiere para la litis en específico.

El anteproyecto también recurre al concepto de interés para facultar una determinada actuación en juicio, siendo posible encontrarlo en los artículos 31 (Designación de curador ad litem); $40 \mathrm{~N}^{\circ} 2$ (Agencia oficiosa); 47 (Intervención voluntaria) en el cual se establece expresamente que el interés actual equivale a tener comprometido un derecho y no una mera expectativa, verificándose una especie de legitimación extraordinaria; 71 (Requisitos de los actos procesales); 387 (Admisión y traslado de la demanda y citación para audiencia de juicio); 389 (Inasistencia de las partes al comparendo); 411 (Impugnación de infracciones legales en el curso de la ejecución); 459 (Convenio de realización judicialmente aprobado); y, 496 (Entrega de cosas genéricas o indeterminadas).

En la generalidad de los casos el documento hace referencia a un interés legítimo y actual y sólo en una norma lo identifica con el hecho de tener efectivamente comprometido un derecho. Si bien es posible comprender la 
Villalobos - Legitimación activa y reforma procesal civil...

finalidad que se ha querido erigir al requerir la efectiva existencia de una posición jurídica amenazada -equiparándose con una especie de interés individual o primario- al analizar la forma en que han sido redactadas las normas, existe el riesgo que ello se satisfaga con la sola afirmación del demandante de la necesidad de protección de una relación jurídica sustancial o que -en el otro extremo- se exija certificar in limine que el actor ha sufrido un perjuicio o dejado de obtener una ganancia (artículo 135).

Sumado a lo anterior, no debe desatenderse la contingencia que trae aparejada la determinación de qué se entiende por interés legítimo, por su constante mutabilidad, al corresponderse simplemente con aquel interés respaldado normativamente. Así las cosas, a través de los artículos citados tampoco se provee una norma de clausura capaz de fundamentar la aceptación o rechazo de tal o cual pretensión, ni se establece un límite que impida la saturación del aparato jurisdiccional.

A su turno, y aunque no se explicita, es posible vislumbrar una vaga exigencia de interés en obrar en el actor, toda vez que deberá -O a lo menos afirmarser el titular del derecho y, como consecuencia de ello, el interesado en protegerlo.

No obstante, conforme se expuso, primeramente resulta forzoso establecer quién posee el interés primario (que proviene de la relación material) para luego enfrentarlo con el demandante que exige tutela jurídica (interés secundario), existiendo la paradoja de que el interés comprometido en el juicio por el cual se pretende la tutela jurídica del interés primario atiende a la relación jurídica procesal por la cual el juez debe decidir y que a la vez dice relación con la situación controvertida de la relación jurídica sustancial sobre la cual el juez deberá pronunciarse.

Expuesta la precedente conclusión es posible sostener que en el anteproyecto el interés primario no fue comprendido ni consagrado como un presupuesto de fundamentación de la LTA, lo que motiva a revisar en el siguiente apartado en qué forma ésta fue entendida y si en su normativización es posible que la misma cumpla con su labor de barrera para aquellas demandas que no podrán ser resueltas con una sentencia que se pronuncie sobre el fondo de la pretensión.

Los artículos de mayor relevancia que se refieren a la LTA son: el artículo 41 (Representación en caso de intereses difusos y colectivos) donde a la vez se recurre al concepto de interés para proveer de legitimación; artículo 62 (Legitimación y oportunidad) por el cual se señala que sólo serán las partes quienes estarán legitimadas para solicitar la acumulación; y, artículo 168 (Objetivo de las medidas prejudiciales y sujetos legitimados para solicitarlas) que subsume la calidad de parte e interés como legitimación.

No obstante la poca metodicidad en construir un solo concepto de LTA, la gran innovación del anteproyecto se encuentra en artículo 248 (Control de 
admisibilidad de la demanda) que permite fallar -incluso antes de que sea trabada la litis - la manifiesta falta de legitimación para actuar, con todas las ventajas que se han expuesto en el presente trabajo; diferenciándola al mismo tiempo de la falta de capacidad o representación; sin perjuicio que en esto último se produce otro inconveniente -planteado supra- sobre la delimitación de la calidad de parte y la (s) capacidad (es) de la (s) misma (s), cuestión que se comprueba con el estudio al artículo citado.

Asimismo, corresponde destacar cómo la misma tendencia se refleja en lo normado para los artículos $258 \mathrm{~N}^{\circ} 9$ (Excepciones previas) y 259 (Efectos de la resolución que se pronuncia sobre excepciones previas), con la salvedad que en el primero de ellos el juez tendrá la tarea de dilucidar las dudas que en forma espontánea se presentan respecto de la efectiva homologación entre el concepto de legitimación e interés por la forma en que se redactó la norma- y la exigencia de que su inexistencia en el demandante deberá surgir manifiestamente de los propios términos de la demanda. En este sentido, el no tener claridad respecto de qué comprende la LTA -al equiparársela, por ejemplo, con la capacidad e interés de manera vacilanterepresentará un desafío para nuestra judicatura al momento de fundar su rechazo a dar curso a una determinada demanda y a la exigencia de presentar o no, y en qué medida, antecedentes que justifiquen la legitimación para demandar.

\section{b) El Proyecto del Nuevo Código Procesal Civil}

En el documento presentado ante la Cámara de Diputados el 18 de mayo del año 2009, se conservan en gran medida las mismas normas que existen en el anteproyecto, pero con algunos cambios en su redacción que no afectan las conclusiones sostenidas preliminarmente.

Es así como respecto del concepto de parte, su capacidad, la capacidad procesal y la ausencia de estas últimas (artículos 19; 20, 21 y 23) el Proyecto resalta el hecho y cualidad de participar en el juicio y el derecho material como fundamento de la capacidad, cuestión que se regula básicamente en el Código Civil, replicándose las mismas conclusiones expuestas en el capítulo precedente.

Con el concepto de interés ocurre prácticamente lo mismo, normándose en los artículos 22 (Designación de curador ad litem); $31 \mathrm{~N}^{\circ} 2$ (Agencia oficiosa); 38 (Intervención voluntaria) conservando la misma posición de que el interés actual equivale a tener comprometido un derecho y no una mera expectativa y aceptando de igual forma una especie de LTA Extraordinaria; 61 (Requisitos de los actos procesales); 315 (Traslado de la demanda y citación a audiencia); 424 (Acuerdo de enajenación); y, 464 (Entrega de cosas genéricas o indeterminadas).

No obstante, en la LTA que se norma principalmente en los artículos: 32 (Representación en caso de intereses difusos y colectivos) donde nuevamente el interés provee de legitimación para demandar; 53 (Legitimación y oportunidad) acudiendo a la calidad de partes como aquella que está legitimada; y 159 (Objetivo de las medidas 
Villalobos - Legitimación activa y reforma procesal civil...

prejudiciales y sujetos legitimados para solicitarlas) que subsume la calidad de parte e interés a la legitimación, es posible verificar algunos cambios normativos.

Si bien el Proyecto conserva el más grande aporte en esta materia, esta vez -en el artículo 236 (Control de admisibilidad de la demanda) - se faculta al mismo Tribunal para establecer, con la sola presentación de la demanda, que ésta no puede ser admitida a tramitación por manifiesta falta de legitimación para actuar declarándolo de plano y expresando los fundamentos en su decisión.

Con lo anterior, el anteproyecto y finalmente el Proyecto, en forma clara y concreta, van más allá de lo que la doctrina nacional evaluaba como un aporte a la reforma, instruyendo a los jueces para desestimar -incluso antes de que se establezcan las partes del proceso; se evalúen las capacidades; y, el interés- que un demandante podría carecer de una manifiesta falta de legitimación para actuar.

Regulado lo anterior en el sentido expuesto, y por la forma en que hasta el momento se encuentra redactada la norma, lo natural es entender que la referencia a la LTA trata de aquella que doctrinariamente se ha analizado a lo largo de esta presentación, cuestión que se confirmaría con la consagración de la excepción que se permite oponer al demandado, al contestar la demanda, en el artículo $245 \mathrm{~N}^{\circ} 9$ (Excepciones previas) que señala: "Excepciones previas. El demandado en la contestación de la demanda puede plantear como excepciones previas: 9. La falta de legitimación o interés". Eliminándose en el Proyecto la frase establecida en el anteproyecto que continuaba diciendo "(...) cuando surja manifiestamente de los propios términos de la demanda".

Del mismo modo es posible identificar un cambio en la regulación del artículo 246 (Efectos de la resolución que se pronuncia sobre excepciones previas), al eliminarse el inciso donde se señalaba que la excepción de falta de legitimación o interés sería resuelta sólo en la medida que el fundamento de la decisión se encontrare debidamente acreditado con los antecedentes existentes ante el Tribunal.

\section{CONCLUSIÓN}

Tanto en el anteproyecto como en el actual Proyecto de Código Procesal Civil, se comprueba una cierta laxitud en el tratamiento de la LTA, particularmente en este último documento, al eliminarse la exigencia de que su ausencia surja en forma manifiesta, y al prescindir de antecedentes que acrediten dicha circunstancia.

La insuficiencia en el tratamiento de la LTA como un concepto unívoco y las divagaciones que se comprueban en el articulado del Proyecto, respecto de cómo se concibe en definitiva a la parte, su capacidad, la capacidad procesal, el interés, cuándo el interés es actual y legítimo, y la legitimación, presentará nuevos desafíos para las comisiones encargadas de examinar el documento. Es de esperar 
REJ - Revista de Estudios de la Justicia - No 14 - Año 2011

que, durante la tramitación legislativa del Proyecto se busque una coherencia conceptual y sistémica entre los conceptos procesales abordados en esta exposición, particularmente respecto al control in limine de la LTA. Su implementación en el sentido propuesto, contribuirá a disminuir sustancialmente los costos públicos y privados de numerosos litigios que, de lo contrario, culminarían con sentencias que se limiten a denegar la acción por no ostentar el demandante la calidad de legitimado. 
Villalobos - Legitimación activa y reforma procesal civil...

\section{BIBLIOGRAFÍA}

* CALAmANDREI, Piero: "Instituciones del Derecho Procesal Civil según el nuevo Código”. Ed. EJEA. Buenos Aires. 1962. En CORDÓN MORENO, Faustino: "Sobre la Legitimación en el Derecho Procesal”. Revista Chilena de Derecho. №2. Vol. 25. Facultad de Derecho Pontificia Universidad Católica de Chile. Santiago. 1998.

* CARnelutTI, Francesco: Instituciones del Proceso Civil. Quinta edición. Ed. Jurídicas Europa-América S.A. Tomo III. Buenos Aires. 1956.

* Couture, Eduardo J.: Fundamentos del Derecho Procesal Civil. Tercera edición. Ed. Depalma. Buenos Aires. 1997.

* DE la BARRA GILI, Francisco: "Responsabilidad extracontractual por daño ambiental: el problema de la legitimación activa". Revista Chilena de Derecho. No2. Vol. 29. Facultad de Derecho Pontificia Universidad Católica de Chile. Santiago. 2002.

* FIGUEROA YÁVAR, Juan Agustín: “¿En que momento procesal puede alegarse la falta de legitimación activa?” Revista de Derecho Procesal. No19. Facultad de Derecho Universidad de Chile. Santiago. 1997.

* GÓMEZ-FERRER MORANT, Rafael: "Derecho a la tutela judicial y posición peculiar de los poderes públicos". Revista Española de Derecho Administrativo. No33. España. 1982.

* GUASP, Jaime: "Derecho Procesal Civil". Ed. Civitas. Madrid. 1998. En MATURANA MIQUEL, Cristián: Disposiciones Comunes a todo Procedimiento. Apuntes de clases. Santiago. Mayo 2003.

* Maturana MIQUel, Cristián: Disposiciones Comunes a todo Procedimiento. Apuntes de clases. Santiago. Mayo 2003.

* MOnRoy GalveZ, Juan: Introducción al Proceso Civil. Ed. Temis. Bogotá. 1996.

* MONTero aroCA, Juan: La Legitimación en el Proceso Civil. Ed. Civitas. Madrid. 1994.

* MOSQUera RUIZ, Mario y MATURANA MIQUEL, Cristian: Breves Nociones acerca de la Cosa Juzgada. Apuntes de clases. Santiago. Mayo 2005.

* Romero SEguEL, Alejandro: "El Control de Oficio de los Presupuestos Procesales y la Cosa Juzgada Aparente. La Capacidad Procesal”. Revista Chilena de Derecho. No4. Vol. 28. Facultad de Derecho Pontificia Universidad Católica de Chile. Santiago. 2001.

* TICONA POSTIGO, Victor: El debido proceso y la demanda civil. Ed. Rodhas. Lima. 1999.

* VESCOVI, Enrique. "Teoría General del Proceso". Ed. Temis. 1984. En MATURANA MIQUEL, Cristián. Disposiciones Comunes a todo Procedimiento. Apuntes de clases. Santiago. Mayo 2003. 